\title{
Usuelle satzwertige Wortverbindungen und gebrauchsbasierte Muster
}

\begin{abstract}
Die Analyse sprachlicher Massendaten zeigt, wie zentral mehr oder weniger feste Wortverbindungen als Lexikoneinheiten für die Sprachkompetenz sind. Der Beitrag geht zunächst kurz auf aktuelle Entwicklungen in der Phraseologie ein: eine Abkehr von der starken Betonung des Idiosynkratischen einerseits und die Hinwendung zum Vorgeprägten andererseits. Wir führen hierzu den Terminus ,lexikalisch geprägte Muster` (LGM) ein. In einer Detailanalyse beschäftigen wir uns mit satzwertigen Wortverbindungen im Spannungsfeld zwischen ausgeprägter Lexikalisierung, die auf eine gesonderte kognitive Verankerung hindeutet, und der Konstitution abstrakter Spruchmuster als LGM-Subtyp. Im zweiten Teil zeigen wir, wie usuelle Wortverbindungen innerhalb von Phrasenkomposita zur Lexembildung beitragen.
\end{abstract}

\section{Einleitung}

Wortverbindungsphänomene rücken zunehmend ins Zentrum anderer linguistischer Disziplinen wie Computerlinguistik, Framesemantik oder kognitive Linguistik (vgl. Schmid 2014). Dies ist ein umso bemerkenswerterer Fakt, als Phraseologisches in der Vergangenheit häufig in die Peripherie linguistischer Theoriebildung verbannt und allenfalls als hübsche Folklore betrachtet wurde, die aus dem Regulären ausschert und deshalb für das Sprachsystem nicht relevant ist. Eine Ausnahme bildeten immer schon die Fremdsprachdidaktik und -lexikografie, die naturgemäß Kollokationen und Phraseme als Schlüssel der Sprachbeherrschung verstehen mussten oder anders herum ausgedrückt: als größte Hürde beim Fremdsprachenerwerb.

Die europäische Phraseologie besitzt jedoch eine lange Tradition, die bis zu Charles Ballys ,Traité de stilistique française‘ 1909 zurückreicht (Burger et al. (Hg.) 2007, S. V). ${ }^{1}$ So ist etwa das Handbuch der Phraseologie von Burger/Buhofer/Sialm (vgl. Burger/Buhofer/Sialm (Hg.) 1982) ein beredtes Zeugnis für die frühe Beschäftigung mit heute so aktuellen Aspekten der Lexikalisierung und Reproduzierbar-

1 Zur Herausbildung der Phraseologie als wissenschaftliche Disziplin vgl. auch ausführlich Fleischer (1997, S. 4-28). 
keit von Mehrwortausdrücken, mit der Frage also, unter welchen Bedingungen eine Abfolge von Wörtern nicht nur eine bloße syntaktische Kombination lexikalischer Elemente, sondern eine autonome sprachliche Einheit darstellt.

In der Phraseologie haben sich in den letzten beiden Jahrzehnten zwei folgenreiche Paradigmenwechsel vollzogen: Der erste betrifft die Liberalisierung des Gegenstandsbereichs, weg von einer starken Fokussierung auf Irreguläres hin zu einem weiten Verständnis von Mehrworteinheiten, zu einer Sicht auf Sprache, die durch ,idiomatische Prägung، (vgl. Feilke 1996) an sich bestimmt ist. Die Erweiterung des Objektbereichs wurde maßgeblich durch die korpuslinguistische Wende getragen, durch die Möglichkeit, auf der Basis sprachlicher Massendaten Strukturen, Muster und Vernetzungen zu erkennen, die sich dem analytischen Blick bis dato verschlossen hatten (vgl. Steyer 2013). Es wurde mehr und mehr deutlich, dass Kollokationen ebenso auf dem Prinzip funktionaler Verfestigung basieren wie Idiome oder jegliche Form vorfabrizierter sprachlicher Äußerungen. Dieser Erkenntnis Rechnung tragend, hat Steyer bereits 2000 den Terminus ,Usuelle Wortverbindungen' (UWV) vorgeschlagen (vgl. Steyer 2000) (siehe Abschn. 2).

Den zweiten Paradigmenwechsel könnte man als „Muster-Wende“ bezeichnen. Die neuen empirischen Erkenntnisse verdeutlichten, dass der sprachliche Normalfall nicht das Okkasionelle, sondern das Vorgeprägte ist (Ágel 2004, S. 67). Und es wurde evident, dass auch mehr oder weniger lexikalisierte Wortverbindungen viel stärker solchen Vorprägungen folgen, als das in der Zeit vor den Korpora erkannt werden konnte. Auch diesbezüglich gab es der Phraseologie bereits früh Denkansätze, beispielsweise zu ,Modellbildungen“ oder ,Phraseoschablonen'. ${ }^{2}$ In letzter Zeit hat besonders die Konstruktionsgrammatik zu dieser MusterWende in der phraseologischen Forschung beigetragen (vgl. Lasch/Ziem (Hg.) 2011; Steyer (Hg.) i.Vorb.).

Die folgenden Ausführungen basieren auf Ergebnissen unserer langjährigen Forschungen, in denen wir korpuslinguistische Vorgehensmodelle entwickelt und für eine musterbasierte Phraseologie(theorie) und deren lexikografische Umsetzung fruchtbar gemacht sowie experimentelle Studien zu neuartigen datennahen Präsentationsformen durchgeführt haben.

2 Vgl. dazu Burger in der mittlerweile fünften und gerade unter diesem Aspekt stark aktualisierten Auflage seiner Einführung in die Phraseologie (Burger 2015). 


\section{UWV und lexikalisch geprägte Muster}

\subsection{Begriffsbestimmung}

UWV sind durch häufigen Gebrauch zu autonomen Mehrworteinheiten geronnen, weil sie als verfestigte Einheit sehr effizient spezifische Aufgaben in der Kommunikation erfüllen. Dies ergibt sich unabhängig davon, ob sie idiomatisch im Sinne der Definition von Burger (2015) oder eher kompositionell sind, welche strukturellen Merkmale sie aufweisen oder ob eine opake Bedeutung etymologisch herleitbar ist. Sie werden häufig fragmentarisch oder verschachtelt verwendet und finden sich in einer komplexen Verflechtung innerhalb des Wortschatzes wieder, etwa mit Monolexemen, wobei nie eine vollkommene Synonymie existiert, sondern immer ein Mehr an Ausdrucksqualität auf der einen oder anderen Seite (sonst würde es nicht beide Lexemtypen geben).

UWV stellen aber auch fast immer Realisierungen unterschiedlich abstrakter Schemata dar und sind in einem Gefüge von anderen Lexemen und Mustern zu verorten. ${ }^{3}$ Wir schlagen hierfür den Terminus , lexikalisch geprägte Muster' (LGM) vor. LGMs sind partiell gefüllte Schemata mit mindestens einer festen lexikalischen Komponente und variablen Slots, deren Füller auch unterschiedlichen morphosyntaktischen Klassen angehören können. Lexikalisch geprägten Mustern können holistische Bedeutungen und/oder Funktionen zugeschrieben werden. Demzufolge gehen wir von einer gesonderten Verankerung im Lexikon aus. Das LGM-Konzept nimmt stärker als ,Konstruktion ${ }^{\circ 4}$ die lexikalische Verfestigung von Syntagmen in den Blick, die nicht immer Realisierungen ein- und derselben syntaktischen Konstruktion darstellen müssen (vgl. Steyer 2013; Steyer i.Vorb.). Wir unterscheiden generell zwischen a) Mustern, deren Slots obligatorisch sind (Wortverbindungsmuster), und b) lexikalischen Erweiterungsmustern. Wortverbindungsmuster können nicht auf den lexikalischen Kern reduziert werden, ohne die eigenständige Bedeutung zu verlieren (z.B. [in ADJ (absehbarer/nächster/...) Zeit] in der Bedeutung ,demnächst‘ vs. *in Zeit). Bei Erweiterungsmustern treten Füller als rekurrente Kotexte fakultativ hinzu und differenzieren den Kern kontextuell aus, (z.B. [mit ADJ (großer/grimmiger/...) Genugtuung]: Kern: mit Genugtuung in der Bedeutung ,positiv konstatierend').

3 Zum schillernden Musterbegriff verweisen wir auf Dürscheid/Schneider (2015).

4 Zum verwandten Terminus, Phrasem-Konstruktion` vgl. Dobrovol'skij (2011). 
UWV und lexikalisch geprägte Muster können formal sowohl unterhalb als auch auf der Satzebene angesiedelt werden (siehe Abschn. 3). ${ }^{5}$

Der entscheidende Punkt ist, dass wir Muster als Ergebnis einer Bottom-upModellierung verstehen. Sie stellen Abstraktionen über rekurrente Syntagmen ähnlicher Art dar, wobei „immer wieder Instanzen einer bestimmten Phrase als Muster (als Vorbilder) für die Produktion weiterer Instanzen dienten“ (Bubenhofer 2009, S. 24).

\subsection{Empirische Methoden}

Solche Musterbildungen sind a priori und regelbasiert kaum vorhersagbar, sondern nur durch eine induktive Korpusmusteranalyse (vgl. auch Hanks 2013) zu rekonstruieren. Unser Vorgehen ist iterativ (von den Daten zu den Hypothesen wieder zu den Daten). Das bedeutet, dass wir jedem Analyseschritt eigene Interpretationen vorschalten und somit mit einem höheren qualitativen Anteil arbeiten als quantitative Ansätze. ${ }^{6}$

Neben iterativen Suchprozessen nach syntagmatischen Einheiten im Korpus stellen die statistische Kookkurrenzanalyse (Belica 1995) als kontextualistischer Zugang zum Gebrauch (vgl. Sinclair 1991) und Slotanalysen auf der Basis von Keyword-in-Context-Zeilen (KWICs) die zentralen Analysemethoden dar. Mithilfe des Analysewerkzeugs LEXPAN ${ }^{7}$ können wir sowohl Kookkurrenzdaten und KWICs qualitativ annotieren und systematisieren, also auch die Slotbesetzungen von Suchmustern untersuchen.

5 Satzwertige Einheiten beziehen sich, so Lüger, auf Sachverhalte und geben Aussagen wieder (sind also Propositionen); bilden relativ abgeschlossene Sinneinheiten; müssen nicht immer syntaktisch vollständig sein, verkörpern aber in jedem Fall funktional vollständige Einheiten (1999, S. 54-57). Einen instruktiven Überblick über Satzkonzepte innerhalb der Phraseologie und in anderen Forschungszusammenhängen gibt Finkbeiner (2008).

6 Unsere primäre Datenbasis ist das Deutsche Referenzkorpus (DeREKo); vgl. Institut für Deutsche Sprache (2017).

7 Das einzelsprachenunabhängige Analyseprogramm LEXPAN wurde in unserem Projekt „Usuelle Wortverbindungen“ zur Unterstützung der linguistischen Interpretation großer Sprachdatenmengen aus Korpora entwickelt. LEXPAN dient der explorativen Untersuchung von Festigkeit, Varianz, Slotbesetzungen und kontextuellen Einbettungsmustern syntagmatischer Strukturen. Es ermöglicht, aus Korpora exportierte Daten in einer eigenen Arbeitsumgebung weiter zu bearbeiten, zu strukturieren und zu kommentieren sowie die Ergebnisse in gesonderten Dateien darzustellen (vgl. LEXPAN). 


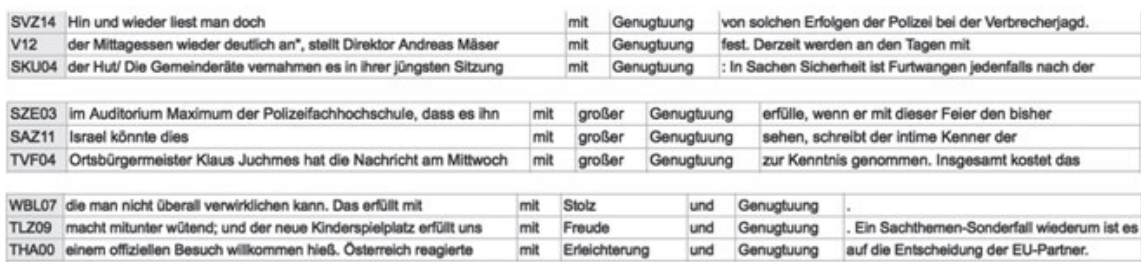

Abb. 1: KWIC-Bündel mit X Genugtuung (LEXPAN: Ausschnitt)

\begin{tabular}{|l|l|l|l|}
\hline \multicolumn{1}{|c|}{ Lückenfüller } & Anzahl & Prozentanteil & Kwics \\
\hline & 7587 & 76,08 & Kwics \\
\hline einen & 1267 & 12,71 & Kwics \\
\hline einen glücklichen & 206 & 2,07 & Kwics \\
\hline puren & 102 & 1,02 & Kwics \\
\hline den & 92 & 0,92 & Kwics \\
\hline einen dummen & 92 & 0,92 & Kwics \\
\hline einen unglücklichen & 65 & 0,65 & Kwics \\
\hline reinen & 64 & 0,64 & Kwics \\
\hline Glück und & 21 & 0,21 & Kwics \\
\hline einen puren & 20 & 0,20 & Kwics \\
\hline diesen & 16 & 0,16 & Kwics \\
\hline glücklichen & 16 & 0,16 & Kwics \\
\hline bloßen & 15 & 0,15 & Kwics \\
\hline irgendeinen & 14 & 0,14 & Kwics \\
\hline einen blöden & 11 & 0,11 & Kwics \\
\hline einen reinen & 11 & 0,11 & Kwics \\
\hline Kommissar & 9 & 0,09 & Kwics \\
\hline Glück oder & 7 & 0,07 & Kwics \\
\hline einen historischen & 7 & 0,07 & Kwics \\
\hline den glücklichen & 6 & 0,06 & Kwics \\
\hline schieren & 6 & 0,06 & Kwics \\
\hline einen schönen & 5 & 0,05 & Kwics \\
\hline einen seltsamen & 5 & 0,05 & Kwics \\
\hline welchen & 5 & 0,05 & Kwics \\
\hline
\end{tabular}

Abb. 2: Automatisch erstellte Lückenfüllertabelle: mit (0-3 Leerstellen) Genugtuung (LEXPAN: Ausschnitt)

Wir konzentrieren uns im Folgenden auf die Analyse satzwertiger Muster und speziell auf so genannte, Spruchmuster‘. Dieser LGM-Typ steht in der Tradition der parömiologischen Forschung. Die Untersuchung von Spruchmustern ermöglicht gleichzeitig aber auch Erkenntnisse, die für aktuelle Forschungsvorhaben zu Fragen der sprachlichen Verfestigung herangezogen werden können. 


\section{Der Satz ist die Botschaft}

\subsection{Satzwertige Musterhaftigkeit}

Werfen wir ein Blick auf folgende drei Beispiele:

(1) Jetzt heißt es Daumen drücken!

(2) Mailand oder Madrid, Hauptsache Italien.

(3) Andere Länder, andere Sitten.

Auf den ersten Blick sind diese Sätze sehr unterschiedlicher Natur, und man würde spontan wahrscheinlich nur (3) einen UWV-Status zuschreiben. Aber bei allen Beispielen handelt es sich um verfestigte, wenn man so will, „lexikalisierte“ Sätze, die eine gewisse Vorkommenshäufigkeit in DEReKo aufweisen. Das Syntagma Jetzt heißt es in (1) stellt eine rekurrente Erweiterung der UWV Daumen drücken dar, und der gesamte Satz hat immerhin eine Häufigkeit von 671 Vorkommen (im Folgenden „V“). Der Satz in (2) ist ein gern zitierter Klassiker aus der Welt der Fußballsprüche (426 V) und bei (3) handelt es sich um ein gebräuchliches deutsches Sprichwort (4.042 V). ${ }^{8}$ Allen Beispielen aber liegen satzwertige Muster zugrunde: (1) [Jetzt heißt es] X; (2) [X Hauptsache $\mathrm{Y}]$ und (3) [Andere $\mathrm{X}$, andere $\mathrm{Y}]$. Betrachten wir zunächst kurz die Muster in (1) und (2).

$\mathrm{Zu}$ (1): Die Analyse der nachgelagerten Slotbesetzungen des Syntagmas Jetzt heißt es ergibt eine auffällige Häufung somatischer Füller, die jeder für sich eine andere Teilbedeutung aufweisen:

(4) Ärmel hochkrempeln:

Nerven/kühlen Kopf bewahren:

Mund abputzen:

Kopf hoch:

Augen zu und durch: ,etwas anpacken

,nicht hektisch werden'

,das Negative schnell vergessen und weitermachen'

,nicht verzagen'

,nicht auf das Schwere, Negative

schauen, sondern durchhalten bzw. weitermachen'

8 DeReKo-Archive: W, W2, W3, W4; COSMAS II-Queries:

(1) (\$jetzt /+w1:1 heißt /+w2:4 Daumen) /s0 drücken; (2) Mailand /+w2:2 Madrid /+w1:3 Hauptsache /+w1:3 Italien;(3) \$andere /+w1:3 Länder /+w1:3 \$andere /+w1:3 Sitten 
Trotz dieser unterschiedlichen Teilbedeutungen instantiieren alle Realisierungen ein übergeordnetes satzwertiges Muster, das funktional restringiert ist: Es wird eine Ermutigung oder Aufforderung ausgedrückt. Diese Illokution wird auch in anderen Vorkommen mit nichtsomatischen, verbalen Ergänzungen realisiert wie Jetzt heißt es \{nach vorn schauen/anpacken/weiter kämpfen/fleißig trainieren\}. Dass es sich hierbei um eine funktionale Gebrauchsbeschränkung eines Musters handelt, verdeutlicht der Vergleich mit anderen Jetzt heißt es-Verwendungen:

(5) 094 Jetzt heißt es wieder überall, die Kärntner SP wird von Wien gegängelt

R98 Jetzt heißt es wieder Schlange stehen auf der verwinkelten Steintreppe

Beispiel (2) ist insofern interessant, als die satzwertige Musterhaftigkeit quasi in einem Monolexem angelegt ist: Das Lexem Hauptsache wird in ca. 50 Prozent der Vorkommen (W: ${ }^{9} 79.410 \mathrm{~V}$ ) in der folgenden Struktur verwendet:

(6) [flexibler Slot Hauptsache flexibler Slot]

Wurscht, Hauptsache gewonnen

Egal, ob die Katze schwarz oder weiß ist, Hauptsache sie fängt Mäuse

Das Aussehen ist für ihn zweitrangig, Hauptsache sie akzeptiert seine glühende Leidenschaft für Bierdeckel

Die holistische Musterfunktion könnte man so umschreiben: ,Unabhängig von der Bewertung eines vorgelagerten Sachverhalts A ist Sachverhalt B positiv zu bewerten'. Ein zweiter häufiger Strukturtyp sind Vorkommen mit Hauptsache am Satzanfang (ca. 35 Prozent des Gesamtvorkommens in W) (z.B. Hauptsache, du bist gesund). Hier wird ein anaphorischer Bezug zu einem im vorausgegangenen Satz ausgedrückten Sachverhalt hergestellt. Dies ändert jedoch nichts an der Musterfunktion. ${ }^{10}$

Die Beispiele (1) und (2) verdeutlichen, dass auch okkasionelle oder gar irrwitzig scheinende Äußerungen auf kognitiven Musterprägungen basieren, und dies ganz unabhängig von der individuellen mentalen Verfassung eines Sprechers.

Das Beispiel (3) zugrunde liegende Muster [Andere X, andere Y] gehört zu den so genannten ,Spruchmustern', auf die wir nun näher eingehen.

9 Die Abkürzung W steht für das DeREKo-Archiv W.

10 Der dritte Verwendungstyp in der Hauptsache (15\%) ist eine textsortengebundene UWV (juristischer Terminus). 


\subsection{Spruchmuster}

Unser Interesse für lexikalisierte Sätze resultiert aus der Arbeit im EU-Projekt „Sprichwort. Eine multilinguale Internetplattform für das Sprachenlernen“ (vgl. SWP; Steyer (Hg.) 2012). ${ }^{11}$ In diesem Kontext haben wir 2.000 Sprichwörter (SW) aus Wörterbüchern und DaF-Lehrwerken mit Hilfe einer iterativen und sehr komplexen Suchheuristik in DeREKo überprüft, ${ }^{12}$ wobei sich das Kriterium der Satzwertigkeit im Sinne von Lüger (siehe oben) für diese Zwecke gut operationalisieren ließ. Zwei Beispiele sollen dies illustrieren: Für den SW-Kandidaten Niemand ist unersetzlich konnte anhand der Korpusbelege eine lexikalisierte Satzwertigkeit nachgewiesen werden:

(7) NUZ10 Für die Partei wäre es eine Zäsur. „Niemand ist unersetzlich“ RHZ01 sagt Wilfried Pauly augenzwinkernd: „Niemand ist unersetzlich, warum sollte ich es also sein?“

NUN15 Niemand ist unersetzlich. Wir haben prima andere Rennen

Der SW-Kandidat Niemand ist ohne Fehl und Tadel kann dagegen nicht als fester Satz angesehen werden:

(8) F95 Wer ohne Fehl und Tadel ist, der werfe den ersten Stein

N92 löste diese Aufgabe ohne Fehl und Tadel

094 die Musik ist ohne Fehl und Tadel

M98 selbst Heilige sind nicht frei von Fehl und Tadel

Die Zwillingsformel Fehl und Tadel (W: 2.243 V) ist zwar hochgradig usuell (fast immer mit der Präposition ohne), aber nur sechsmal mit niemand. Keines dieser Vorkommen weist jedoch eine lexikalisierte Satzstruktur auf. Damit ist Niemand ist ohne Fehl und Tadel kein fest geprägter Satz.

Unsere Korpuserhebung hat gezeigt, dass Sprecher wenig bis gar nicht zwischen Sprichwort, geflügeltem Wort, Slogan und dergleichen unterscheiden, dass sie sehr wohl aber ein ausgeprägtes Bewusstsein von festen Sätzen und ihren Funktionen haben. Wenn sprachliche Einheiten beispielsweise als Sprichwort gekennzeichnet werden, dann realisieren diese Ausdrücke zumeist auch die typischen

11 Der von uns erarbeitete deutsche Wörterbuchteil wurde nach Abschluss des EU-Projekts als „Sprichwörterbuch“ in OWID überführt und seither modular ausgebaut (vgl. SWB).

12 Ca. 900 waren belegt, davon ca. 500 in seriösen Frequenzbereichen (die häufigsten liegen bei 3.000 bis 4.000 Vorkommen und mehr). 
Spruch-Funktionen in kondensierter Form wie das Vermitteln von Alltagsurteilen oder -erfahrungen bzw. Illokutionen wie Warnung, Mahnung, Aufforderung. Im Folgenden verwenden wir deshalb den Terminus ,Spruch' als Oberbegriff (vgl. Fix 2007), und für lexikalisch partiell gefüllte Sprucheinheiten ,Spruchmuster‘.

In der traditionsreichen Parömiologie wurden Spruchmuster bereits vielfach diskutiert, etwa als ,proverbial patterns‘, ,proverb formula‘, ,proverb frames‘, ,Bauformen‘ oder ,Denkschablonen‘ (vgl. z.B. Taylor 1934; Röhrich/Mieder 1977). Spruchmuster finden sich im OWID-Wörterbuch in großer Zahl, wie die folgenden Beispiele zeigen (in Klammern jeweils ein prototypisches Sprichwort):

(9) [Was X, (das) Y] (Was lange währt, wird gut)

[Wenn X, (dann) Y] (Wenn es dem Esel zu wohl wird, geht er aufs Eis)

[Wer X, (der) Y] (Wer sucht, der findet)

[Wie X, so Y] (Wie der Vater, so der Sohn)

[Wo X ist, ist (auch) Y] (Wo ein Wille ist, ist auch ein Weg)

[Erst X, dann Y] (Erst die Arbeit, dann das Vergnügen)

[Kein X ohne Y] (Kein Nachteil ohne Vorteil)

Spruchmuster kann man auf verschiedenen Abstraktionsebenen ansiedeln, von Mustern, die durch die lexikalischen Komponenten stark aufgeladen werden und bei denen die Slots im Grunde kontextuelle Modifikationen desselben Spruchs darstellen wie Übung macht den \{Meister/Handballmeister/Champion/Virtuosen\}, bis hin zu relativ abstrakten Mustern, die von Strukturformeln gesteuert werden und dementsprechend ein breites Spektrum an Ausfüllungen bis in den nichtspruchhaften Bereich aufweisen. Dies diskutieren wir jetzt an den SW-Beispielen Andere Länder, andere Sitten [Andere X, andere Y] und Besser spät als nie [Besser X als Y].

\subsection{Gebrauchsrestriktionen bei Spruchmustern}

Das Spruchmuster [Andere X, andere Y] weist allein in W eine Frequenz von 5.283 auf ${ }^{13}$ und lässt sich wie folgt paraphrasieren: ,Wenn etwas vom Eigenen oder Geltenden abweicht, dann weicht auch das Dazugehörende ab‘. Aufgrund dieser Bedeutung könnte man sich produktive Ausfüllungen jeglicher Art vorstellen, wie man auch an bestimmten Ad-hoc-Vorkommen sieht, z.B. Andere Produkte, andere Preise oder Andere Ärzte, andere Diagnosen. Die Füllertabelle zeigt daneben aber auch folgendes Bild:

13 Query: \$andere /+w2:2 andere 


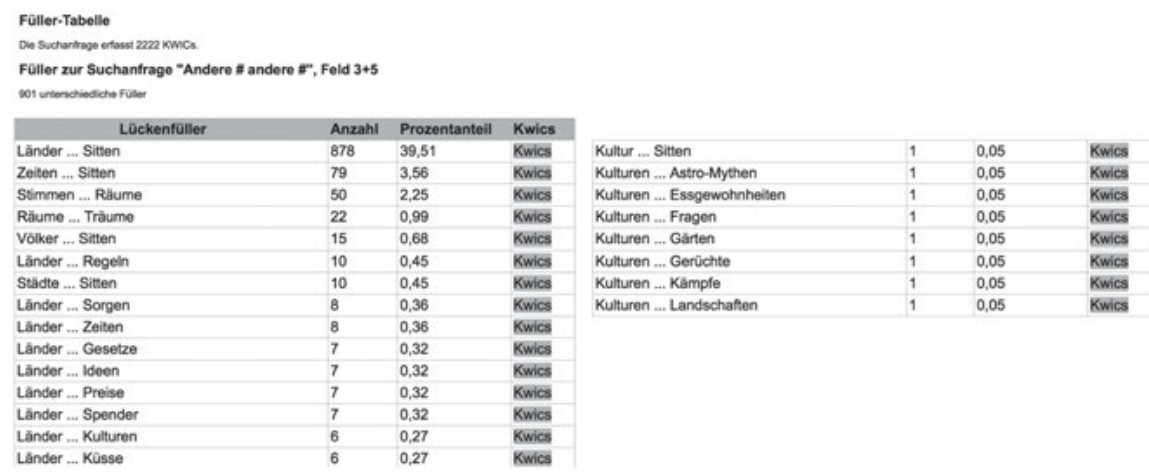

Abb. 3: Automatisch erstellte Lückenfüllertabelle Andere X, andere Y (LEXPAN: Ausschnitt)

Es wird zum einen ersichtlich, dass das Sprichwort Andere Länder, andere Sitten mit knapp 40 Prozent die prototypische Realisierung ist und in knapp 60 Prozent eine Vielzahl anderer Realisierungen zu verzeichnen ist. Die überproportional häufige sprichwörtliche Realisierung ist kein Einzelfall, sondern kann für fast alle Spruchmuster beobachtet werden. Dies deutet ganz klar auf eine separate kognitive Verankerung von Sprichwörtern hin (siehe Abschn. 5). Aber auch bei den anderen Füllern liegt eine Präferenz vor: Ein Großteil der Füller referiert auf Konzepte wie NATIONALITÄT - KULTUR - VERHALTEN, was ebenso im Bereich der Füller untermauert wird, die nur einmal belegt und als Ad-hoc-Vorkommen anzusehen sind. Möglicherweise ist hier das Sprichwort prägend für das Muster; dem müsste man allerdings in musteretymologischen empirischen Untersuchungen nachgehen.

Das zweite Beispiel ist das Spruchmuster [Besser X als Y]. Bereits Seiler (1922, S. 191) sieht besser - als als häufigste Komparativformel des Deutschen an. Er nimmt u.a. die folgende Klassifikation typischer Füllungen vor, die wir ebenso im Korpus nachweisen konnten:

a) Substantive: Besser Hammer als Amboss;

b) Adjektive und Partizipien: Besser arm in Ehren als reich in Schanden;

c) Infinitive: Besser betteln als stehlen;

d) Sätze: Besser es fresse mich ein Wolf als ein Schaf

Die Slotanalyse ergibt ein breites Spektrum an Füllergruppen und Konzepten: In ca. 30 bis 35 Prozent realisieren die Füllungen ein temporales Konzept, die prototypische Realisierung (22 Prozent) ist Besser spät als nie. Andere temporale Verwendungen sind Besser heute als morgen/jetzt als nie/früher als später bzw. das SW Besser ein Ende mit Schrecken als ein Schrecken ohne Ende. 
Die zweite signifikante Füllergruppe stellen Wortpaare dar wie die Folgenden:

(10) besser gemeinsam als einsam; aktiv als passiv

overdressed als underdressed

besser vorbeugen als heilen; verwerten als verbrennen

besser Prävention als Repression; Minijobs als Schwarzarbeit

Diese Wortpaare sind teilweise im echten Sinne antonymisch (wie gemeinsam einsam), teilweise drücken sie aber nur stark diskurs-kontextabhängige Präferenzrelationen aus (wie Minijob - Schwarzarbeit).

Eine dritte Füllergruppe umfasst Syntagmen wie diese:

(11) Besser den Spatz in der Hand als die Taube auf dem Dach

Besser ein Ende mit Schrecken als ein Schrecken ohne Ende

Besser mehrere dünne Schichten übereinanderstreifen als wenige dicke

Hier ist interessant, dass einerseits wieder Sprichwörter, andererseits aber auch nichtsprichwörtliche Feststellungen Instantiierungen darstellen. Sprecher bedienen sich des Spruchmusters, um eine Aussage gleichsam als Erfahrungserkenntnis zu positionieren wie beim Beispiel Besser mehrere dünne Schichten übereinanderstreifen als wenige dicke.

Die Realisierungen des [Besser $\mathrm{X}$ als $\mathrm{Y}$ ]-Musters weisen einen unterschiedlichen Grad an semantischer Eigenständigkeit auf: Während Sätze wie Besser vorbeugen als heilen oder Besser ein Ende mit Schrecken als ein Schrecken ohne Ende tatsächlich allein als Erfahrungssätze stehen können, ist dies bei vielen anderen Beispielen nicht so eindeutig der Fall. Besser früher als später ist zwar semantisch noch relativ autonom, erfordert aber eine stärkere kontextuelle Anbindung (Was soll besser früher als später geschehen?).

Die folgenden beiden Beispiele illustrieren, wie sich der autonome Status ganz auflöst und in den Text verlagert:

(12) Bei den Azzurri stimmten bislang nur die Ergebnisse. Besser so als umgekehrt - siehe Spanien. (DEREKo, Hamburger Morgenpost, 30.6.2006, S. 2)

(13) „Über die Bezahlung rede ich nicht. Aber ansonsten ist der Job ok. Es macht Spaß. Besser jedenfalls, als Papier und Müll von den Straßen zu sammeln, was andere Ein-Euro-Jobber tun müssen [...]“ (DEREKo, Nordkurier, 11.5.2010) 
Hier ist die Präferenzrelation eines Sachverhalts gegenüber einem anderen nicht mehr allein in den elliptischen Besser-als-Sätzen inkorporiert, sondern wird wiederum erst durch die Referenz auf eine Proposition im Satz davor hergestellt. Trotz dieses graduellen autonomen Status der Realisierungen lässt sich aber mit Permjakov eine gemeinsame abstrakte Musterbedeutung für alle Realisierungen formulieren:

Wenn eine Sache eine bestimmte Eigenschaft hat, und eine andere Sache hat eine andere Eigenschaft oder dieselbe zu einem unterschiedlichen Grad, so ist eine der Sachen der anderen vorzuziehen. (Permjakov 2000, S. 75)

[Gruppe 22: Qualitative Überlegenheit $\leftrightarrow$ Unterlegenheit von Sachen]

Neben dieser abstrakten Bedeutung kann man diesem Muster in vielen Fällen eine zusätzliche - graduelle - Konnotation zuschreiben: ,X ist ZWAR AUCH NICHT perfekt, ABER IMMER NOCH positiver als $Y^{‘}$. Die holistischen Musterfunktionen sind immer Absicherung, Rat oder Mahnung.

\section{4 (Folgenlose) Spruchtransformationen}

Das dritte Beispiel beruht auf dem Satz Wer zu spät kommt, den bestraft das Leben, der zu einem neuen deutschen Sprichwort geworden ist. Über den Prozess der Ersetzung des eigentlich am 6.10.1989 in damaligen Ostberlin von Michail Gorbatschow geäußerten Satzes Gefahren warten nur auf jene, die nicht auf das Leben reagieren, ist viel geschrieben worden (z.B. Mieder 2010). Aber um diese Genese geht es hier nicht. In unserem Argumentationszusammenhang ist interessant, dass die lexikalische Komplettersetzung (bis auf das Wort Leben) an dem Konsequenz-Topos (vgl. dazu Wirrer 2007) und an der abstrakten Bedeutung nichts geändert hat: ,WENN jemand etwas Bestimmtes tut/unterlässt, DANN hat das für ihn negative Folgen'. Bei der Ersetzung des ursprünglichen Satzes wurde die klassische Wer-der-Struktur aktualisiert, ${ }^{14}$ die dann wiederum das Weiterleben dieses Spruchs und die neuerliche Musterbildung ermöglicht hat. Interessant ist nun der Verweis Mieders auf das Sprichwörter-Lexikon von Friedrich Wilhelm Wander (Hg.) (1876), der bereits einige Wer zu spät kommt-Sätze auflistet (Sp. 667):

14 Eine prototypische SW-Realisierung ist z.B. Wer rastet, der rostet. Auch hier kann man das Kontinuum bis in den nichtsprichwörtlichen Bereich verfolgen bei Vorkommen wie: Wer nicht hundertprozentig bei der Sache ist, (der) baut den Gegner auf. 
53 Wer spät kommt, dem bleiben die Knochen. Dän.: Forsømmelse tager hvad hende bydes, og rekker efter meere. (Prov. dan., 184.)

54 Wer spät kommt, der kommt auch.

55 Wer spät kommt, der wohnt schlecht. It.: Chi tardi arriva, mal alloggia. (Pazzaglia, 369, 1.)

56 Wer spat kompt, der sitze hinder der Thür. - Lehmann, II, 852, 340; Suringar, CLXXXIII, 6; Körte, 5970.

It.: Chi tarde arriva, male alloggia.

Lat.: Sero venientes male sedentes.

Schwed.: Den sist kommer, får sämsts säte. (Marin, 8.)

57 Wer spat komt gesessen, der muss vbel essen.

- Henisch, 1558, 69; Suringar, CXVIII, 2.

Dän.: Hvo til gilde kommer silde, han skal sidde ilde, og æde ilde. (Prov. dan., 231.)

58 Wer zu spät kommt, esse mit den Gemalten an der Wand. - Körte, 5623; Simrock, 9664; Suringar, 78, 2.

59 Wer zu spät kommt, findet leere Schüsseln.

Holl.: Die te laat komt, vindt den schotel omgekeerd. (Harrebomée, $I I, 260^{\mathrm{a}}$.)

60 Wer zu spät kommt, hat das Nachessen (Nachsehen). - Eiselein, 482; Simrock, 7270; Braun, I, 2870.

Abb. 4: Auszug aus dem Artikel „Spät“ des Deutschen Sprichwörter-Lexikons

Nun ist nicht davon auszugehen, dass die Reformulierer den Spruch in den Wirren jener Wende-Tage 1989 im Wander nachgeschlagen haben. Aber augenscheinlich gibt es unterschiedliche kognitiv verfestigte Inventare, derer Sprecher sich bewusst oder unbewusst je nach kommunikativer Aufgabe bedienen: In unserem Fall des Wer-der-Musters und des Konzepts des Zu-Spät-Kommens als Mahnung, etwas zu verpassen und negative Folgen zu erleiden.

In Abschnitt 4 wollen wir abschließend zeigen, inwieweit diese Muster-Mechanismen auch bei einem Wortbildungstyp, den Phrasenkomposita, greifen.

\section{Feste Sätze in Phrasenkomposita}

Wortverbindungen können nicht nur Lexemstatus haben, sondern innerhalb komplexer morphologischer Strukturen wiederum selbst zur Lexembildung und Musterkonstitution beitragen: Sie sind als "frozen syntactic fragments“ (Toman 1985, S. 411) in Phrasenkomposita (PKs) wie Die-Zukunft-kann-mich-mal-Handy (siehe Abb. 5) integrierbar: 


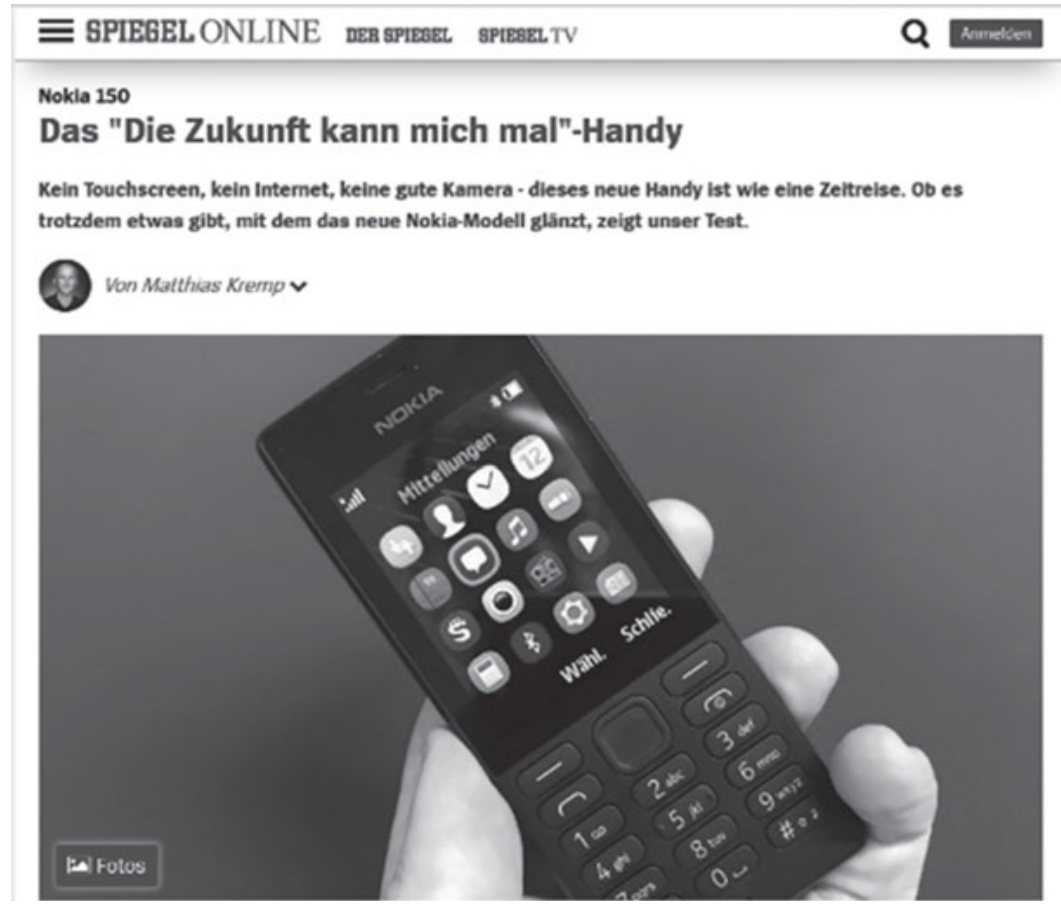

Abb. 5: Beschreibung des Nokia 150 (www.spiegel.de/netzwelt/gadgets/nokia-150-im-test-dasdie-zukunft-kann-mich-mal-handy-a-1133990.html)

\subsection{Was sind Phrasenkomposita?}

Bei PKs handelt es sich um Komposita, deren „Erstglied bzw. [...] Bestimmungswort keine lexikalische Kategorie, sondern eine phrasale Komponente [...]“ ist (Lawrenz 2006, S. 7), d.h. eine syntaktische Phrase oder ein Satz (vgl. u.a. Hein 2015, 2017; Meibauer 2003; Trips 2016). Anhand einer Bildung wie „Geiz ist geil“-Parole lässt sich exemplarisch illustrieren, dass PKs prinzipiell genauso funktionieren wie prototypische Determinativkomposita (z.B. Baumhaus): Die Bildung ist in zwei Unmittelbare Konstituenten zerlegbar, zwischen denen ein hierarchisches Verhältnis (grammatische und semantische Dominanz des Zweitglieds) besteht: [,Geiz ist geil“ $]_{\text {Erstglied }}[\text { Parole }]_{\text {Zweitglied }}$. 


\subsection{Perspektiven auf sprachliche Verfestigung}

Die Spielarten sprachlicher Verfestigung, die sich in PKs manifestieren, lassen sich aus unterschiedlichen Perspektiven betrachten.

Zum einen kann die Phrasenkomposition - wie in der konstruktionsgrammatisch ausgerichteten Modellierung in Hein (2015) - ausgehend von den Eigenschaften der Grundwörter durch die Annahme von Konstruktionen auf unterschiedlichen Abstraktionsebenen bottom up systematisiert werden. ${ }^{15}$

Zum anderen lassen sich PKs ausgehend von den Wortverbindungen (WV) in Erstgliedposition als Fall sprachlicher Verfestigung betrachten und in das UWVModell integrieren. Unter Einnahme dieser Perspektive steht im Folgenden die Frage im Mittelpunkt, welche Funktion satzwertige Einheiten wie Geiz ist geil in PKs wie „Geiz ist geil “-Parole ${ }^{16}$ haben. Die Datenbasis bildet das auf DEREKo basierende PK-Korpus aus Hein (2015), das insgesamt 1.576 substantivische PK-Types umfasst. Die Subgruppe der PKs mit satzwertigem Erstglied macht ca. 22 Prozent dieses Untersuchungskorpus aus; sie weist gegenüber den PKs mit satzgliedwertigem Erstglied (78 Prozent des Untersuchungskorpus, z.B. 5-TageWoche, Heile-Welt-Gerede) einen erhöhten Expressivitätsgrad auf. ${ }^{17}$

\subsection{Typen von festen Sätzen}

Im Folgenden wird exemplarisch aufgezeigt, dass sich innerhalb der satzwertigen PK-Erstglieder das gesamte Kontinuum zwischen lexikalisierten WVs und Realisierungen abstrakter WV-Muster manifestiert, das wir im Rahmen unseres UWV-Modells rekonstruiert haben.

15 Vgl. Hein (2015) für eine ausführliche empirische Untersuchung (u.a. Untersuchungskorpus, Analysekategorien) und eine theoretische Modellierung der Phrasenkomposition im Rahmen der Konstruktionsgrammatik.

16 Alle PK-Beispiele stammen aus Hein (2015) und werden in ihrer Originalschreibung aus DEREKo wiedergegeben (z.B. Gebrauch von Bindestrichen, Art und Position der Anführungszeichen).

17 Dies wird auch durch den Vergleich der Type-Token-Relation untermauert: Der TTR-Wert der Gruppe der PKs mit satzwertigem Erstglied $(0,77)$ ist deutlich höher als der TTR-Wert der Gruppe der PKs mit satzgliedwertigem Erstglied (0,08). Dies deutet auf eine hohe Anzahl von Hapaxen/ Ad-hoc-Bildungen innerhalb der hier fokussierten Subgruppe hin. 
Satzwertigkeit wird in den phrasalen Erstgliedern formal durch ganz unterschiedliche Typen, d.h. durch WVs unterschiedlichen syntaktischen Komplexitätsgrades, realisiert: ${ }^{18}$

(14) Vollsätze

- „Wir sitzen alle in einem Boot“-Gerede

- „Jetzt geht's los“-Motto

(15) Elliptische Sätze

- Schwach-wie-Flasche-leer-Rede

- ,Jetzt erst recht!'-Parole

(16) Elliptische Satzschemata ${ }^{19}$

- Sowohl-als-auch-Verhalten

- Einerseits-andererseits-Geschwafel

(17) Syntaktische Phrasen

(,selbstständige Phrasenstrukturen‘, vgl. Finkbeiner 2008)

- „Freie Fahrt“-Ruf

-, „Ab in den Süden“-Motto ${ }^{20}$

\subsubsection{Lexikalisierte satzwertige Wortverbindungen}

In Erstgliedposition von PKs finden sich zahlreiche lexikalisierte Spruch-WVs. Die Übergänge zwischen einzelnen Spruchtextsorten wie z.B. Slogan oder Sprichwort sowie die Bewusstheit der Autorschaft sind hier - wie an anderer Stelle schon als Charakteristikum von UWV herausgestellt - oft fließend.

Um klassische Sprichwörter handelt es sich bei den PK-Erstgliedern des Untersuchungskorpus interessanterweise nur sehr selten (18). Häufig vertreten sind vielmehr zahlreiche WVs aus dem Übergangsbereich zwischen Sprichwort und Slogan (19-20):

18 Auch in Bezug auf die Grundwörter, die durch satzwertige PK-Erstglieder bevorzugt modifiziert werden, lassen sich Tendenzen erkennen (z.B. Grundwörter wie Motto, die einen Standpunkt wiedergeben), eine gezielte empirische Überprüfung steht jedoch noch aus.

19 Diese Bildungen sind insofern ,schematisch', als sie nur paarige Konnektoren enthalten, aber keine zu verknüpfenden Inhalte (vgl. strukturelle Phraseologismen, Burger 2015).

20 Das Erstglied $A b$ in den Süden zeigt, inwiefern der expressivere Subtyp der Phasenkomposition auch spezifische kommunikative Funktionen (hier: appellativ, direktiver Sprechakt) übernehmen kann. 
(18)

Wo-gehobelt-wird-fallen-Späne-Aspekt

Tatsächlich ist das Interessanteste und gleichzeitig Bedrückendste an diesem Brief aber gar nicht der Wo-gehobelt-wird-fallen-Späne-Aspekt seines Arguments für den Amoklauf (DEREKo, die tageszeitung, 22.11.2006, S. 13)

(20)
Weniger-ist-mehr-Motto; „Less is more“-Credo

Geiz-ist-geil-\{Verhalten/Parole/Devise/Aspekt/Motto\}

Das Erstglied Geiz-ist-geil (20) tritt musterhaft im Untersuchungskorpus auf; seine beobachtbare Kombinierbarkeit mit verschiedenen substantivischen Grundwörtern lässt sich wiederum bottom up durch die Ansetzung eines abstrakten Musters beschreiben: [Geiz-ist-geil-X]. Ursprünglich als erfolgreicher Werbeslogan der Elektronikkette Saturn geprägt, lässt sich für diesen Spruch mittlerweile eine abstraktere, d.h. breitere Verwendung - und somit die Entwicklung in Richtung Sprichwort - konstatieren. ${ }^{21}$

Ein weiterer verbreiteter Erstgliedtyp sind außerdem Sprüche aus dem Bereich Politik, bei denen die Autorschaft noch sehr präsent ist, vgl. z.B. das berühmte Zitat Martin Luther Kings in (21):

$$
\text { „I have a dream"-Rede }
$$

„Ich bin ein Berliner-Rede“

„The Medium is the message“-Gerede

,America-first ${ }^{*}$-Credo

Bei der Erstgliedbildung von PKs vermehrt genutzt werden außerdem Zitate aus dem Bereich Sport. Dies wird im Folgenden (25-27) exemplarisch durch den Rückgriff auf diverse PKs rund um Giovanni Trapattonis legendäre Rede auf einer Pressekonferenz im Jahr 1998 illustriert. Diese Erstglieder sind auch insofern interessant, als es sich durchweg um grammatisch nicht wohlgeformte Syntagmen handelt - ihrer Integration in ein Wortbildungsprodukt steht dies aber offenbar nicht im Weg.

$$
\text { "Was-erlauben-Strunz } " \text {-Rede }
$$

$$
\underline{\text { Schwach-wie-Flasche-leer-Rede }}
$$

21 Die abstrakte Bedeutung von Geiz ist geil wird in unserem OWID-Sprichwörterbuch wie folgt beschrieben: „Sagt man dafür, dass es positiv ist und Freude macht, möglichst wenig Kosten für etwas aufzuwenden“ (www.owid.de/artikel/404075; vgl. Steyer/Polajnar 2014). 
(27) Ich-habe-fertig-Rede: Oder der FC Bayern München ohne die tragikomischen Inszenierungen seines Personals, das dem Fußball-Branchenführer einst den Beinamen FC Hollywood eingebracht hat? Man denke nur an die legendäre Ich-habe-fertig-Rede des Fußball-Maestros Giovanni Trapattoni [...]. (DEREKo, Frankfurter Allgemeine, 7.7.1999)

\subsubsection{Stereotype}

Neben diesen lexikalisierten WVs ist ein Erstglied-Typ identifizierbar (28), der zwar an der sprachlichen Oberfläche nicht oder nicht zwingend verfestigt ist, dem aber bestimmte konzeptuelle Muster bzw. Stereotype zugrunde liegen. Die Auflösung von Gesamtkomplexen mit solchen WVs ist dementsprechend nicht ohne den Einbezug von Weltwissen möglich.

„Der tut nichts"-Hund

Bekannte mußten vor wenigen Tagen ihren sechs Jahre alten Dackel einschläfern lassen. Der ist beim Spazierengehen von solch einem „Der tut nichts“-Hund gebissen worden. (DEREKo, Rhein-Zeitung, 4.12.1998)

Das - auch strukturell verfestigte - Erstglied in „Der tut nichts“-Hund evoziert eine bestimmte Szene: Es handelt sich um eine typische Aussage von Hundebesitzern, wenn ihr Hund außer Kontrolle geraten ist und umstehende Passanten davon überzeugt werden sollen, dass keine Gefahr von dem freilaufenden Hund ausgeht.

In (29) und (30) sind weitere Beispiele für PKs angegeben, in denen mit solchen Stereotypen gespielt wird. Versprachlicht werden Letztere durch die Integration von „original or fictional quotation“ (Finkbeiner/Meibauer 2016, S. 39; vgl. auch Pascual/Królak/Janssen 2013).

„die Gabi fand das aber gar nicht schön“-Softie

„Wir-haben-alles-besser-gemacht“-Geplapper

Solche PKs haben eine stark kondensierende Funktion; sie spielen indirekt auf stereotype, charakteristische Eigenschaften eines Personentypus und dessen klischeehaften Verhaltensweisen an. ${ }^{22}$

22 In (29) wird der spezifizierte Personentypus direkt im Grundwort genannt (,Softie`), während in (30) durch die Beschreibung typischer Argumentationsstrategien (,Geplapper') eine indirekte Spezifizierung des Personentypus ,Politiker' vorgenommen wird. 
Entsprechende WVs werden auch genutzt, um konkrete Personen (also nicht nur Personen-Typen) indirekt, aber markant zu umschreiben - ein Paradebeispiel im Untersuchungskorpus sind PK-Bildungen rund um Franz Beckenbauer (31-33):

Ich-kann-Golf-und-Schi-und-Wandern-und-bin-schöner-als-die-andernFranz

Ich-kanns-Franz

Wo-ich-bin-ist-vorne-Franz

Die Funktion der Zitate im Erstglied ist mit der Funktion vergleichbar, die die eingeschobenen Zitate in so genannten ,komplexen Personennamen-Konstruktionen (middle-name constructions) vom Typ [N „CP“ $\mathrm{N}$ ] übernehmen (Finkbeiner/Meibauer 2016), beispielsweise Boris „Ich bin drin“ Becker oder Oliver „Ich will den Erfolg sofort" Kahn.

\subsubsection{Satzwertige WV-Muster}

Neben lexikalisierten WVs und konzeptuellen Mustern handelt es sich bei vielen Erstgliedern zugleich auch um lexikalische Realisierungen abstrakter (satzwertiger) WV-Muster. Zwei Prämissen sind in diesem Zusammenhang zentral: Erstens ist der Musterstatus primär extern begründet, d.h. er hängt nicht davon ab, wie usuell ein bestimmtes Muster für die Phrasenkomposition ist. Zweitens werden WV-Muster in PKs - wie im Folgenden noch ersichtlich wird - in ganz unterschiedlichem Maße realisiert.

Zunächst zu einem Muster, das bei der Erstglied-Bildung von PKs sehr frequent ist und somit auch durch die Phrasenkomposition selbst konstituiert wird:

$$
\text { [X }\{\text { oder/und/statt }\} \text { Y] }
$$

Bei den Instantiierungen dieses Musters handelt es sich um ,phraseologische Wortpaare‘ (vgl. Fleischer 1997) bzw. „Paarformeln', d.h. um Verbindungen von „zwei Wörter[n] der gleichen Wortart oder auch zweimal dasselbe Wort [...]“ (Burger 2015, S. 55). Diese Wortpaare werden im Untersuchungskorpus entweder implizit oder explizit, d.h. mit Hilfe von Konjunktionen (und, oder, statt), verknüpft:

(35) $\quad$ Friß-oder-stirb-Rede; „Zu mir oder zu dir“-Gequatsche; $\underline{\text { Alles-oder-Nichts- }}$ Devise 
(36) Ora-et-labora-Woche; Raus-und-Weg-Bedürfnis; Pleiten-Pech-und-PannenWoche

(37) „Freiheit statt Sozialismus“-Parole; Versöhnen-statt-spalten-Rede; Kinderstatt-Inder-Parole

(38) Veni-Vidi-Vici-Devise; Friede-Freude-Eierkuchen-Parole ${ }^{23}$

Aber auch in Fällen, die innerhalb der PKs nicht produktiv sind, kommt Musterhaftigkeit ins Spiel: Das abstrakte WV-Muster in (39) wird in Erstgliedposition nur

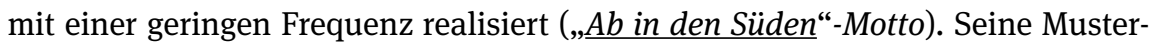
haftigkeit ist extern begründet, d.h. sie konstituiert sich durch seine frequente Realisierung außerhalb morphologischer Strukturen:

[Ab in ART SUBST]

In der Forschungsliteratur wurde das Muster [Ab in ART SUBST] (außerhalb von PKs) bereits als produktive ,Adv-PP-Direktiv-Konstruktion' erfasst (mit Realisierungen wie $A b$ durch die Mitte (Finkbeiner 2008) oder Raus aus meinem Haus! (Jacobs 2008)). Zudem ist die extern begründete Musterhaftigkeit auch in DeReKo nachweisbar; besonders frequente Realisierungen sind etwa $A b$ in \{den Urlaub/ den Süden/die Wüste/die Ferien/...\}.

\section{Resümee}

Wir konnten anhand sprachlicher Spuren in Massendaten zeigen, dass Sprecher auf unterschiedliche Inventare verfestigter lexikalischer Mehrworteinheiten und lexikalisch geprägter Muster zurückgreifen, und zwar:

a) verfestigte Wortverbindungen (z.B. Geiz-ist-geil-X),

b) teilspezifizierte Muster (z.B. [Andere $\mathrm{X}$, andere $\mathrm{Y}]$ ),

c) eher abstrakte Strukturmuster (z.B. [Wer X, der Y]).

Die Entscheidung, welches Inventar in welchen Teilstrukturen mit welchen Füllungen zu aktivieren ist, ist abhängig vom kommunikativen Ziel. In der Regel haben wir es mit Mehrfacheinträgen im Lexikon zu tun, als verfestigte lexikalische Exemplare und als Musterrealisierungen. Die LGM-Slots werden am oberen

23 Zur Diskussion des Phrasenstatus solcher implizit verknüpfter Wortpaare vgl. Hein (2015, S. 194 f.). 
Pol der Häufigkeitsskala häufig durch prototypische Füller besetzt (bei Spruchmustern z.B. Sprichwörter), aber auch Ad-hoc-Bildungen folgen in systematischer Weise diesen Vorprägungen. Sowohl bei Spruchmustern als auch in PKs mit satzwertigem Erstglied liegt ein Kontinuum vor, von stark lexikalisierten, autonomen UWV, die für sich genommen auch kontextfrei funktionieren, bis hin zu Einheiten, die nur durch die textuelle Einbettung decodiert werden können.

Eine systematische Untersuchung solcher Musterbildungen kann nicht nur für die Erhellung kognitiver Mechanismen sprachlicher Verfestigung gewinnbringend sein, sondern eröffnet auch Perspektiven für eine neuartige Musterlexikografie und -didaktik. Diese basiert auf der grundlegenden Unterscheidung zwischen lexikalisierten Wortverbindungen, die man aufgrund ihres Verfestigungsgrads quasi wie Vokabeln lernen muss, und lexikalisch geprägten Mustern, bei denen man die Bildung verstehen muss.

\section{Internetquellen}

Institut für Deutsche Sprache (2017): Deutsches Referenzkorpus/Archiv der Korpora geschriebener Gegenwartssprache 2017-II (Release vom 1.10.2017). Mannheim. Internet: www.ids-mannheim.de/DeReKo (Stand: 24.10.2017).

LEXPAN: Lexical Pattern Analyzer (Version 2017-04-13). Ein Analysewerkzeug zur Untersuchung syntagmatischer Strukturen auf der Basis von Korpusdaten. Entwickelt vom Projekt „Usuelle Wortverbindungen“, Institut für Deutsche Sprache, Mannheim. Internet: www1. ids-mannheim.de/lexik/uwv/lexpan.html (Stand: 15.7.2017).

SWB: Sprichwörterbuch in OWID. Internet: www.owid.de/wb/sprw/start.html (Stand: 24.10.2017).

SWP: EU-Sprichwortplattform. Internet: www.sprichwort-plattform.org (Stand: 24.10.2017).

\section{Literatur}

Ágel, Vilmos (2004): Phraseologismus als (valenz)syntaktischer Normalfall. In: Steyer, Kathrin (Hg.): Wortverbindungen - mehr oder weniger fest. (= Jahrbuch des Instituts für Deutsche Sprache 2003). Berlin/New York, S. 65-86.

Belica, Cyril (1995): Statistische Kollokationsanalyse und Clustering. Korpuslinguistische Analysemethode. Mannheim.

Bubenhofer, Noah (2009): Sprachgebrauchsmuster. Korpuslinguistik als Methode der Diskursund Kulturanalyse. (= Sprache und Wissen 4). Berlin u.a.

Burger, Harald (2015): Phraseologie. Eine Einführung am Beispiel des Deutschen. 5., neu bearb. Aufl. Berlin.

Burger, Harald/Buhofer, Annelies/Sialm, Ambros (Hg.) (1982): Handbuch der Phraseologie. Berlin u.a. 
Burger, Harald et al. (Hg.) (2007): Phraseologie. Ein internationales Handbuch der zeitgenössischen Forschung. Bd. 1. (= Handbücher zur Sprach- und Kommunikationswissenschaft 28.1). Berlin u.a.

Dobrovol'skij, Dmitrij (2011): Phraseologie und Konstruktionsgrammatik. In: Lasch/Ziem (Hg.), S. 111-130.

Dürscheid, Christa/Schneider, Jan Georg (2015): Handbuch Satz, Äußerung, Schema. (= Handbücher Sprachwissen 4). Berlin/Boston.

Feilke, Helmuth (1996): Sprache als soziale Gestalt. Ausdruck, Prägung und die Ordnung der sprachlichen Typik. Frankfurt a.M.

Fleischer, Wolfgang (1997): Phraseologie der deutschen Gegenwartssprache. 2., durchges. u. erg. Aufl. Tübingen.

Finkbeiner, Rita (2008): Idiomatische Sätze im Deutschen. Syntaktische, semantische und pragmatische Studien und Untersuchung ihrer Produktivität. Stockholm.

Finkbeiner, Rita/Meibauer, Jörg (2016): Boris ,Ich bin drin' Becker (,Boris I am in Becker`). Syntax, semantics and pragmatics of a special naming construction“. In: Lingua 181, S. 36-57.

Fix, Ulla (2007): Der Spruch - Slogans und andere Spruchtextsorten. In: Burger et al. (Hg.), S. 459-468.

Hanks, Patrick (2013): Lexical analysis. Norms and exploitations. Cambridge, MA.

Hein, Katrin (2015): Phrasenkomposita im Deutschen. Empirische Untersuchung und konstruktionsgrammatische Modellierung. (= Studien zur Deutschen Sprache 67). Tübingen.

Hein, Katrin (2017): Modeling the properties of German phrasal compounds within a usagebased constructional approach. In: Trips, Carola/Kornfilt, Jaklin (Hg.): Further investigations into the nature of phrasal compounding. Berlin, S. 119-148.

Jacobs, Joachim (2008): Wozu Konstruktionen? In: Linguistische Berichte 213, S. 3-44.

Lasch, Alexander/Ziem, Alexander (Hg.) (2011): Konstruktionsgrammatik III. Aktuelle Fragen und Lösungsansätze. (= Stauffenburg Linguistik 58). Tübingen.

Lawrenz, Birgit (2006): Moderne deutsche Wortbildung. Phrasale Wortbildung im Deutschen. Linguistische Untersuchung und sprachdidaktische Behandlung. (= Schriftenreihe Philologia 91). Hamburg.

Lüger, Heinz-Helmut (1999): Satzwertige Phraseologismen. Eine pragmalinguistische Untersuchung. Wien.

Meibauer, Jörg (2003): Phrasenkomposita zwischen Wortsyntax und Lexikon. In: Zeitschrift für Sprachwissenschaft 22, 2, S. 153-188.

Mieder, Wolfgang (2010): „Spruchschlösser (ab)bauen“. Sprichwörter, Antisprichwörter und Lehnsprichwörter in Literatur und Medien. Wien.

Pascual, Esther/Królak, Emilia/Janssen, Theo A.J.M. (2013): Direct speech compounds: Evoking socio-cultural scenarios through fictive interaction. In: Cognitive Linguistics 24, 2, S. 345-366.

Permjakov, Gregorij L. (2000): Die Grammatik der sprichwörtlichen Weisheit. In: Grzybek, Peter (Hg.): Die Grammatik der sprichwörtlichen Weisheit von G.L. Permjakov. Mit einer Analyse allgemein bekannter deutscher Sprichwörter. (= Phraseologie und Parömiologie 4). Baltmannsweiler, S. 43-104.

Röhrich, Lutz/Mieder, Wolfgang (1977): Sprichwort. (= Sammlung Metzler 154). Stuttgart.

Schmid, Hans-Jörg (2014): Lexico-grammatical patterns, pragmatic associations and discourse frequency. In: Herbst, Thomas/Schmid, Hans-Jörg/Faulhaber, Susen (Hg.): Constructions, collocations, patterns. (= Trends in Linguistics. Studies and Monographs 282). Berlin/ Boston, S. 239-293. 
Seiler, Friedrich (1922): Deutsche Sprichwörterkunde. (= Handbuch des deutschen Unterrichts an höheren Schulen 4/3). München.

Sinclair, John (1991): Corpus, concordance, collocation. Oxford.

Steyer, Kathrin (2000): Usuelle Wortverbindungen des Deutschen. Linguistisches Konzept und lexikografische Möglichkeiten. In: Deutsche Sprache 28, S. 101-125.

Steyer, Kathrin (Hg.) (2012): Sprichwörter multilingual. Theoretische, empirische und angewandte Aspekte der modernen Parömiologie. (= Studien zur Deutschen Sprache 60). Tübingen.

Steyer, Kathrin (2013): Usuelle Wortverbindungen. Zentrale Muster des Sprachgebrauchs aus korpusanalytischer Sicht. (= Studien zur Deutschen Sprache 65). Tübingen.

Steyer, Kathrin (Hg.) (i.Vorb.): Sprachliche Verfestigung. Wortverbindungen, Muster, Phrasemkonstruktionen. (= Studien zur Deutschen Sprache 79). Tübingen.

Steyer, Kathrin (i.Vorb.): Lexikalisch geprägte Muster - Modell, Methoden und Formen der Onlinepräsentation. In: Steyer (Hg.) (i.Vorb.).

Steyer, Kathrin/Polajnar, Janja (2014): Werbesloagans. Modul des Sprichwörterbuchs in OWID. Internet: http://owid.de/wb/sprw/start.html (Stand: 9.11.2017).

Taylor, Archer (1934): Problems in the study of proverbs. In: Journal of American Folklore 47, 183, S. 1-21.

Toman, Jindřich (1985): A discussion of coordination and word syntax. In: Toman, Jindřich (Hg.): Studies in German grammar. Dordrecht u.a., S. 407-432.

Trips, Carola (2016): An analysis of phrasal compounds in the model of parallel architecture. In: ten Hacken, Pius (Hg.): The semantics of compounding. Cambridge, S. 153-177.

Wander, Karl Friedrich Wilhelm (Hg.) (1867): Deutsches Sprichwörter-Lexikon. Ein Hausschatz für das deutsche Volk. Bd. 4. Leipzig.

Wirrer, Jan (2007): Phraseme in der Argumentation. In: Burger et al. (Hg.), S. 175-187. 